\title{
'Nuevos' valores en la práctica psicosocial y comunitaria: Autonomía compartida, auto-cuidado, desarrollo humano, empoderamiento y justicia social*
}

'New' Values in the Psychosocial and Community Practice: Shared Autonomy, Self-care, Human Development, Empowerment and Social Justice

Enviado: $1^{\circ}$ de marzo de 2015 | Revisado: $1^{\circ}$ de junio de 2015 | Aceptado: $1^{\circ}$ de agosto de 2015

\author{
ALIPIO SÁNCHEZ VIDAL ** \\ Universidad de Barcelona, España
}

doi:10.11144/Javeriana.up14-4.nvpp

Para citar este artículo: Sánchez, A. (2015). 'Nuevos' valores en la práctica psicosocial y comunitaria: Autonomía compartida, auto-cuidado, desarrollo humano, empoderamiento y justicia social. Universitas Psychologica, 14(4), 1235-1244. http://dx.doi.org/10.11144/Javeriana.up14-4.nvpp

* Artículo de reflexión

**anchezvi@ub.edu

\section{RES UMEN}

Los valores son concepciones de lo bueno que iluminan analíticamente y guían prácticamente la acción humana. Con meritorias excepciones la psicología comunitaria ha descuidado explicitar y discutir abiertamente sus dimensiones éticas y valorativas. Con el fin de remediar parcialmente ese descuido, propongo en este artículo algunos valores y enfoques valorativos novedosos en la práctica comunitaria. Sugiero primero modificaciones para adecuar los valores deontológicos -pensados para la clínica- a la mayor complejidad y dinamismo del trabajo comunitario. Propongo así sustituir la autonomía individual por una autonomía compartida que extienda la auto-dirección al conjunto de la comunidad. Introduzco también el auto-cuidado (auto-beneficio legítimo) para garantizar la integridad psicológica y moral del practicante y la sostenibilidad del trabajo comunitario. Resalto después tres valores socio-comunitarios. El desarrollo humano que junto a la auto-dirección incluye la interacción personal y la vinculación social. El empoderamiento, un valor instrumental fruto de la conciencia subjetiva, la comunicación y la acción social eficaz. Y lajusticia social (valor finalista junto al desarrollo humano) compuesto por un mínimo vital humano (universal), la distribución equitativa de los bienes y recursos materiales y psicosociales producidos por la sociedad, y la relación igualitaria con los demás. Palabras clave

valores; psicología comunitaria; ética; autonomía compartida; auto-cuidado; desarrollo humano; empoderamienteo; justicia social

\begin{abstract}
A B S T R A C T
Values are conceptions of good which enlighten and guide human analysis and action. Discounting noteworthy exceptions, community psychology has neglected making explicit and openly discussing its ethical and value dimensions. My aim in this paper to partially remedy such neglect by posing new sustantive values and approaches suitable for community practice. I suggest first changes in the deontological values to adapt them to the complexity and dynamism of community work. So I put forward shared or collective autonomy, that extends self-direction to the whole community, to substitue for individual disolving autonomy. I also introduce self-care (legitimate self-beneficence) to guarantee psychological and moral integrity of the practitioner as well as long term sustainability of communiy action. I describe, secondly, some core communitarian values. Human development which includes interaction and social bonding besides self-direction. Empowerment, an instrumental value, made of subjective consciousness, communication, and effective social action. Social justice, the main socio-communitarian value, consist of three components:
\end{abstract}


a vital universal minimum, fair distribution of material and psychosocial goods and resources produced by society, and igualitarian personal treatment and relationship.

Keywords

values; community psychology; ethics; shared autonomy; self-care; human development; empowerment; social justice

"Obra de tal modo que trates a la humanidad en tu propia persona y en la de los demás siempre y al mismo tiempo como un fin, nunca solo como un medio"

Emmanuel Kant

Lapsicología comunitaria (PC) no ha valorado sus implicaciones valorativas y éticas que solo a partir de la década de 1990, comienzan a recibir la atención explícita que por su importancia merecen. Aunque ya en 1977 Rappaport señaló en el subtítulo de su libro a los valores como uno de los tres pilares ("valores, investigación y acción”) que sostienen el campo, este ha centrado sus esfuerzos en la acción trasformadora y la investigación, relegando a un segundo plano la identificación y fundación de valores, así como la construcción de una ética práctica diferenciada de la deontología clínica y de la retórica académica o congresual. Pero cualquiera que sea su mérito intrínseco, ni la regla deontológica ni el gran discurso éticopolítico son apropiados para la práctica comunitaria, lo cual genera respectivamente, abordajes individuales no aptos para la complejidad y el dinamismo de los asuntos comunitarios y las generalidades retóricas interesantes para el debate intelectual pero de escasa utilidad como guía de análisis y práctica concretos. Es justo, sin embargo, señalar algunas excepciones a la desatención general de los valores y la ética en el campo comunitario y psicosocial: Bermant, Kelman, \& Warwick, 1978; Heller, 1989; Jeger \& Slotnick, 1982; Kofkin, 2003; Laue \& Cormick, 1978; Montero 2004; Nelson \& Prilleltensky, 2005; O’Neill, 1989; Prilleltensky, 1997; 2001; Sánchez, 1999; 2004; 2007; en prensa; Serrano, 1994; Snow, Grady, \& Goyette-Ewing, 2000; Winkler Alvear, Olivares, \& Pasmanik, 2012.

No estoy negando la vocación ética inherente a la empresa comunitaria en sus distintas versiones, sino el esfuerzo sostenido, visible y específico por concretar y hacer explícita esa vocación mediante la discusión pública de las distintas posturas valorativas y prácticas que con frecuencia se dan por 'conocidas' y acordadas. Sostengo que el esfuerzo dedicado a la ética y los valores no resiste la comparación -en términos cualitativos o cuantitativos- con aquel dedicado en la PC a otras áreas como la descripción de programas y experiencias o la investigación empírica. Tampoco el número de referencias citadas (que podrían ampliarse considerablemente) refuta la tesis: el descuido ético no es cuestión de cantidad sino de cualidad y valoración. Eso es visible en: 1) el limitado valor reconocido a las temáticas éticas y valorativas en relación a su crucial importancia en la práctica y la teoría comunitarias; 2) la calidad periférica o adjetiva reconocida a esos temas, a menudo vistos, no como temas inherentemente valiosos, sino como meros acompañantes (las 'cuestiones éticas' planteadas por el asunto o acción X) de otros temas -técnicos, empíricos o teóricos-sustantivos que suelen llenar las revistas, libros y ponencias.

\section{Causas y efectos}

El examen del discurso y la literatura escrita permite identificar (Davidson, 1989; Kofkin, 2003; Sánchez, 2004) cuatro razones distintas pero de efectos convergentes, para explicar la relegación valorativa y la desatención ética: el excesivo activismo en detrimento de los fundamentos valorativos (y teóricos) de la acción; la debilidad de la base científica y técnica del campo que aumenta la incertidumbre y la dificultad para prever y controlar los efectos de la acción, lo que dificulta la asunción de responsabilidades; el influjo del positivismo racionalista que aconseja prescindir de los valores para poder construir una ciencia 'dura' respetable y una práctica neutral; y el 'buenismo' maniqueo, puesto que nos mueven las mejores intenciones y tratamos de hacer el bien, ipor qué preocuparse por explicitar nuestros valores y de los efectos reales de nuestras acciones?

El descuido explícito de los valores, la insuficiencia de la elaboración sistemática y explícita de una ética comunitaria y el injustificable sentimiento de superioridad moral han tenido como 
efecto una extensa anomia práctica que el psicólogo comunitario ha paliado echando mano de los limitados valores deontológicos existentes o los ha 'absorbido' experimentando una sensación de fracaso y un estrés tan inmerecidos como difíciles de evitar. El abordaje explícito y sistemático de una ética psicosocial y de los valores apropiados a las aspiraciones y características del trabajo comunitario es por tanto, una tarea teóricamente relevante y prácticamente urgente.

Como parte de esa tarea, presento en este artículo 'nuevos' valores de la práctica psicosocial y comunitaria. La novedad consiste en explicitar operativamente valores socio-comunitarios básicos (como la justicia social), redefinir valores deontológicos -como la autonomía- tradicionalmente vistos como atributos individuales, para adecuarlos al trabajo comunitario e introducir valores ausentes de las propuestas al uso, como el auto-cuidado. Me baso para ello en Bermant, et al. (1978), los artículos de O'Neill (1989), Heller (1989) y otros en el monográfico del American Journal of Community Psychology, así como mis escritos anteriores, sobre todo los de 1999; 2007 y en prensa. Antes de describir los nuevos enfoques y valores defino brevemente los valores morales y sus funciones de la acción comunitaria.

\section{Sistemas de valores morales y acción comunitaria}

Los valores morales encarnan concepciones de lo humanamente bueno (como la justicia, la autonomía o la verdad) en las acciones y relaciones humanas y en sus consecuencias. Asumo que el bien y el mal no son absolutos sino que -como los valores que los representan- admiten graduación de forma que podemos valorar una acción o relación $\mathrm{A}-\mathrm{y}$ sus consecuencias-como mejor o más deseable moralmente que una acción o relación B. En la práctica podemos concebir los valores morales como cualidades deseables de las personas (autonomía, veracidad) o la sociedad y sus instituciones (justicia social, solidaridad, reciprocidad) cuyo conjunto limitado forma el ideal concreto de persona, comunidad o sociedad, el 'perfil moral' personal que desearíamos para no- sotros mismos (o nuestros hijos), o social, deseable para la comunidad en que querríamos vivir.

¿Qué función tienen los valores en PC? ¿Cuál es su relación con la actuación del practicante? $\mathrm{Al}$ ser los valores los que dibujan cualidades personales (valores morales personales) o sociales (valores morales sociales) deseables, el practicante debe promoverlos explícitamente, pero también implícitamente en sus actitudes, relaciones y actuación profesional. De tal forma, su relación con los clientes o la comunidad ha de ser veraz, equitativa y respetuosa con los otros y su actuación social ha de contribuir a aumentar la justicia social, la solidaridad o reciprocidad de la comunidad. Los valores éticos iluminan en resumen, los análisis, y guían la conducta del practicante comunitario al identificar las características de la sociedad en que queremos vivir y de las personas con las que merece la pena convivir.

El manejo práctico de los valores en la acción social demanda una visión totalizadora en que, lejos de ser algo absoluto, con una 'valía' inmanente y fija, los valores han de formar sistemas o constelaciones de valía acordada que la gente -personas o instituciones sociales- tiende a asociar e interrelacionar. Por otro lado, como los practicantes no tienen el tiempo, la capacidad ni los medios precisos para promover todos los valores a la vez y en el mismo grado, la gestión práctica de los valores en PC exige jerarquizarlos según su valía en un contexto y coyuntura subjetiva y examinar las relaciones entre los diversos valores relevantes según su contenido y significado concreto en la situación dada. Un paso inicial de la gestión práctica de los valores en PC será, por tanto, identificar qué valores y en qué medida son relevantes para los sujetos en la situación abordada.

Nótese que las anteriores consideraciones prácticas no niegan la universalidad de ciertos valores acordados como tal (Doyal y Gough, 1994), sino el absolutismo práctico de cualquier valor singular y su constancia evaluativa. Niega en otras palabras, que un valor determinado tenga siempre y en cualquier lugar, un mérito moral fijo e invariable, independiente de las circunstancias personales y el contexto social. Así, la justicia social no tendrá la misma importancia y fuerza movilizadora para las personas 
en una situación de gran desigualdad y privación que en una sociedad más uniformemente rica y con mínimas diferencias. No significa lo mismo la libertad para una persona encarcelada o viviendo en una dictadura que para otra que vive en una sociedad con las libertades básicas garantizadas (y las necesidades vitales cubiertas).

\section{Valores deontológicos en psicología comunitaria}

Aunque los valores y principios deontológicos (Beauchamp \& Childress, 1999; França-Tarragó, 1996) tienen una base clínica y están pensados para guiar la actuación profesional con clientes individuales, conciernen a temas éticos básicos de la acción humana y gozan de un amplio consenso profesional. Recogen tres valores asociados a los actores centrales de la acción profesional: la autonomía del cliente, su beneficencia y la justicia social, el valor de la sociedad. A ellos debemos añadir la veracidad y la confianza-soportes de la relación entre profesional y cliente- y el interés propio -segundo valor del profesional-para tener un conjunto coherente. Pero para poder usar de manera fructífera esos valores en PC es preciso modificarlos haciendo una lectura más social y apropiada de las condiciones, aspiraciones y forma de trabajar comunitaria.

Así, en el caso de beneficencia -de significado claro con clientes individuales-, en presencia de diversos actores sociales hay que preguntarse iqué beneficencia - $\mathrm{O}$ no maleficencia- debe primar en caso de conflicto o de desigual distribución de los efectos positivos y negativos de una opción dada? Por otro lado, la confianza está pensada para relaciones diádicas entre dos individuos pero, icómo cambia su definición y cultivo práctico en la relación de un practicante (o un equipo) con grupos y colectivos sociales?, iqué hacer cuando una forma de actuar o relacionarnos fortalece la confianza con un grupo A pero debilita la que mantenemos con $\mathrm{B}$ o C? Asimismo, la veracidad puede tener una lectura inicial como veracidad factual, sencilla y aceptable en situaciones simples y bien definidas: decir la verdad sobre datos, hechos y conductas verificables. Pero, icómo la definimos en relaciones y temas sociales complejos que siempre incluyen un gran caudal de subjetividad y diversidad? En ese supuesto, a los datos y hechos objetivos hay que añadir el efecto de la subjetividad individual (significados, sentimientos, valores) y los intereses sociales que permitan captar la visión particular de cada sujeto (las 'verdades parciales') y a su vez, el grado de integración o coherencia global final de los datos objetivos y subjetivos, la veracidad holística. El concepto de justicia social usado en la deontología es en fin, demasiado estrecho (supone poner a disposición de todos los bienes y técnicas psicológicas y no discriminar a los clientes) y será ampliado en el apartado de valores socio-comunitarios.

Me centro ahora en las dos 'novedades' valorativas más importantes en este apartado: la extensión de la autonomía (generalmente leída como valor o atributo individual) y la reivindicación del autocuidado como una forma de auto-interés legítimo del practicante.

\section{Autonomía colectiva o compartida}

Podemos definir la autonomía como la capacidad (suma de libertad y poder) de auto-determinación personal, de crear y realizar un proyecto de vida propio. La adopción de este valor, generalmente entendido como autonomía individual, para guiar la práctica comunitaria genera un doble problema: 1) la autonomía, elevada a la categoría de valor social supremo -como sucede en el Occidente moderno- fomenta el individualismo y el egoísmo ético y erosiona seriamente la solidaridad, deviene (Bellah, Madsen, Sullivan, Swidler, \& Tipton, 1989) un peligroso disolvente social; 2) al ser un atributo de titularidad individual, resulta inadecuado para una actuación centrada en el colectivo comunitario. Parece preciso por tanto, modificar y ampliar el contenido de la autonomía individual al ámbito colectivo para que como autonomía compartida sea compatible con el desarrollo humano del conjunto de la comunidad, apropiada para la PC.

Esa nueva concepción ampliada de la autonomía debería -en mi opinión- cumplir dos condiciones: 1) trascender la meta de liberación de relaciones u opresiones destructoras (autonomía de), incluyendo y subrayando la meta positiva de desarrollar las 
potencialidades de personas y grupos (autonomía para); 2) reconocer la reciprocidad e interdependencia personal y ser compatible con el ejercicio de una solidaridad que permita recrear la comunidad y realizar proyectos colectivos compartidos. Esa combinación de autonomía positiva y solidaridad (que llamaríamos autonomía compartida o colectiva) permitiría el desarrollo, tanto de la individualidad y los proyectos personales como de la colaboración social en pos de objetivos compartidos o negociados que trascienden las metas estrictamente individuales. Entiendo que en caso de conflicto entre la autonomía de algunos individuos particulares y la global de la comunidad, primaría en principio, la de la comunidad.

Aun cuando la autonomía colectiva propuesta es más apropiada para la PC y resuelve varias de las objeciones hechas a la concepción individual del valor, no llega a ser óptima. Plantea, al contrario, retos que pueden desbordar el mandato social y capacidades del campo (Doyal y Gough, 1994; Sen, 1990) dado que implica la construcción de un conjunto de instituciones y sistemas sociales capaces de ofrecer las opciones colectivas que permitan a las comunidades y personas concretas elegir -dentro de ciertos límites- una forma de vivir significativa y valiosa.

\section{Interés propio y auto-cuidado}

Modificando la propuesta de Thompson (1989) conviene añadir también el interés propio del practicante, un valor ligado al cuidado de sí mismo y entendido más como un suelo ético para sostener psicológicamente al practicante o como un principio modulador (o limitador) de los otros y no como un valor orientador básico. El mandato de auto-cuidado (y el valor de self-interest en el que se

\section{CUADRO 1.}

Autonomía personal y compartida o colectiva

- Promover la capacidad de auto-determinación (tomar decisiones y dirigir la propia vida) de personas y comunidades.

- Tratar a personas y/o comunidades como agentes de sus propias decisiones y acciones y no como objeto de las acciones, intenciones o fines del practicante.

- Respetar la dignidad y capacidad de elegir, decidir y actuar por sí mismo.

- Pactar con el destinatario los fines de la acción y obtener su consentimiento voluntario e informado.

- Informar sobre la acción a realizar, derechos y deberes mutuos y consecuencias previsibles.

- Evitar relaciones y situaciones sociales que creen dependencia, desamparo o impotencia.

- Evitar intervencionismo: no hacer por los demás lo que estos puedan hacer por sí mismos.

- Reconocer y recordar la necesidad de compartir con otros la capacidad de decisión y auto-

determinación en asuntos de titularidad colectiva.

- Recordar la necesidad y conveniencia de participar en las decisiones y acciones colectivas que promueven la autodirección compartida.

- Facilitar la interacción y vinculación social que permiten definir y perseguir el bien común.

Fuente: elaboración propia

CuADro 2.

Auto-interés legítimo, auto-cuidado

\footnotetext{
- 'Suelo' ético: marca límites humanos a otros principios; permite al practicante trabajar eficaz y sostenidamente para otros.

- Cuidarse a sí mismo: el practicante es también sujeto, no solo medio técnico para el bien de la comunidad.

- Derecho a mantener la integridad física y psicológica.

- Derecho a no ser utilizado por el otro y a no implicarse afectiva o personalmente en su vida y problemas más allá de lo exigido por la beneficencia general y la buena praxis.

- Derecho a los medios (información, motivación y colaboración, medios económicos, etc.) precisos para alcanzar los fines pactados.

- Derecho a mantener la reputación profesional y a condiciones de trabajo dignas.
}

Fuente: elaboración propia 
fundamenta) derivan de la extensión al practicante de la cualidad de sujeto ético -no de mero medio técnico para el bienestar del cliente-y de la consiguiente aplicación del principio de no maleficencia que le asegura la misma protección de la que gozan otros sujetos de la acción profesional o comunitaria. Estamos así reafirmando la universalidad ética en el área profesional.

Pero el auto-cuidado cumple también una importante función práctica: evitar que el practicante pueda ser utilizado por la comunidad más allá de lo psicológicamente saludable (y moralmente aceptable), lo que produciría la 'quema' (burn out) del profesional y la consiguiente ineficacia e insostenibilidad de su actuación en el tiempo. Poner límites psicológicos a la disponibilidad del practicante es, en resumen, una exigencia ética y estratégica relevante para proteger personalmente al practicante y para garantizar su competencia y beneficencia profesional a la larga. El auto-cuidado reconocería al practicante (Cuadro 2) ciertos derechos básicos como mantener su integridad, no ser utilizado por el otro para sus propios fines (más allá de lo pactado y moralmente admisible), o los derechos a los medios precisos para alcanzar fines acordados y a la reputación profesional.

\section{Valores y principios socio-comunitarios}

Como cualidades sociales deseables, los valores socio-comunitarios deben ser más apropiados para iluminar analíticamente y orientar prácticamente la PC. Dado que su emergencia es reciente y no han sido suficientemente explicitados y discutidos, ignoramos el grado de consenso profesional y aceptación social del que distintas propuestas -como la que aquí se esboza- podrían gozar. Enunciemos -antes de concretar su contenido- dos características adicionales de estos valores. Una, que al ser valores y principios sociales, conciernen a toda la sociedad (o comunidad), que es la responsable final de su realización; el practicante es solo parcialmente responsable (co-responsable) de promoverlos. Dos, como en los valores personales, podemos hacer una distinción -significativa pero no absoluta- entre valores finalistas -que al tener una 'valía' moral intrínseca marcan los fines de la acción- y valores instrumentales, cuyo mérito moral depende de la medida en que, como medios o métodos, promueven los valores finalistas. Así, la participación sería un valor instrumental para el empoderamiento que a su vez será instrumental para los fines de justicia social y desarrollo humano (valores finalistas).

El sistema socio-comunitario de valores y principios propuesto gira en torno a la justicia social y la solidaridad e incluye el compromiso y la responsabilidad social, la diversidad, el desarrollo humano, el empoderamiento, la participación y organización social, y la eficacia. Describo ahora tres valores novedosos o relevantes para la $\mathrm{PC}$-desarrollo humano, empoderamiento y justicia social-centrándome sobre todo en el último como principal valor 'construido' del sistema socio-comunitario (siendo la solidaridad el valor 'natural' central).

\section{Desarrollo humano personal y colectivo}

El desarrollo humano (Cuadro 3) puede entenderse de forma individual (desarrollo de las personas) o colectivo (desarrollo de la comunidad o la sociedad). Se refiere al despliegue armónico de las capacidades potenciales (personales o comunitarias) en interacción dinámica con el entorno material y psicosocial bajo la dirección del sujeto. No solo implica auto-dirección personal o colectiva (autonomía), sino también, vinculación social, equilibrio -entre distintos aspectos y capacidades- e integración de esos aspectos en un proceso unitario gobernado por el sujeto. En vista de los estragos sociales y morales causados por el exceso de autonomía individual (y su asociación con el egoísmo ético y la utilidad económica) y de sus insuficiencias en la acción comunitaria, estoy proponiendo el desarrollo humano como valor moral alternativo tanto para el trabajo individual como micro-social, comunitario ¿Por qué? Porque como valor (y concepto) más amplio y abarcador, no solo presupone el desarrollo humano, la auto-dirección personal o colectiva (el aspecto aceptable de la autonomía), sino también, inter-acción personal y vinculación social como claves constructivas del desarrollo de la humanidad (individual y colectiva) y de los procesos de toma de decisiones y actuación de los sujetos que conducen a su logro. Es- 
CuAdro 3.

Desarrollo humano

- Promover el despliegue integral y auto-dirigido de las potencialidades y capacidades personales en interacción dinámica con un contexto socio-cultural que lo facilite.

- Apoyar la posibilidad de las personas de crear y realizar un proyecto de vida propio.

- Ayudar a crear contextos psicosociales (familias, grupos, etc.) que faciliten el desarrollo humano y desanimen relaciones y estructuras innecesariamente represivas o coartadoras.

- Ampliar la gama de opciones socio-económicas y culturales entre las que la gente puede elegir efectiva y responsa-

blemente lo que puede ser o hacer (la vida que quieren llevar).

- Desarrollo humano = 'suma' de auto-dirección decisoria o vital, relación interpersonal y cooperación social, igualdad de acceso a, y la distribución de, medios socio-políticos y jurídicos (participación política, seguridad e igualdad jurídica, igualdad de oportunidades sociales...)

Fuente: elaboración propia

CUADRO 4.

Empoderamiento

- Adquisición de poder personal participando en acciones colectivas para alcanzar bienes y

metas sociales valiosos y legítimos.

- Impulsar el sentimiento subjetivo de potencia (sobre todo en grupos marginales), generando expectativas de empoderamiento.

- Promover/fortalecer los procesos de comunicación social que posibilitan la elaboración de objetivos comunes, la organización social y la participación en acciones colectivas.

- Ayudar a discriminar los contextos sociales que permiten el empoderamiento cooperativo de aquéllos en que hay que usar el conflicto y la confrontación para redistribuir el poder.

- Asesorar/facilitar la realización de acciones eficaces en la consecución de bienes sociales valiosos que permiten la adquisición efectiva de poder y confirman las expectativas iniciales.

Fuente: elaboración propia

tos supuestos hacen al concepto de desarrollo humano particularmente apto para una PC que combina la asunción de auto-determinación humana con la causalidad social (en el doble plano de interacción personal e influencia socio-institucional).

\section{Empoderamiento}

El empoderamiento (empowerment) es el proceso de adquisición de poder y su resultado sustantivo, el poder efectivamente conseguido. Es un valor instrumental para los fines de desarrollo humano (que exige libertad de elección y poder de realización) y justicia social que involucra la equidad en la distribución de poder y recursos sociales y psicológicos a partir de un mínimo vital básico. Operativamente el empoderamiento implica (Sánchez, 2013) la conciencia subjetiva de poder, la comunicación entre personas y grupos, y la organización y participación de los sujetos en acciones eficaces para conseguir recursos sociales valiosos, bien sea por medio de la cooperación -que permite compartir el poder con otros-, o a través del conflicto para redistribuir el poder. Por eso el empoderamiento es un valor procedimental clave para la justicia social al fortalecer las capacidades intrínsecas de las personas y comunidades para el desarrollo humano (Cuadro 4).

\section{Justicia social: Justicia distributiva, mínimo vital y equidad relacional}

$\mathrm{Al}$ ser un valor de titularidad social, la definición y realización de la justicia social compete a toda la sociedad; los actores y sectores sociales específicos tienen papeles parciales (idealmente complementarios y convergentes) en su logro en función de los aportes (educativos, económicos, psicológicos, etc.) de cada sector y de la contribución que la correcta gestión de esos aportes puede hacer a la justicia social global. Como valor cardinal de cualquier sociedad (Rawls, 1971), esta ha sido concebida y operativamente definida de diversas maneras. Desarrollo aquí los tres 
componentes identificados por Bellah et al. (1989) que son pertinentes para guiar la acción comunitaria dirigida a promover ese valor: un componente sustantivo, 'de mínimos'; la redistribución de bienes y recursos sociales; y el componente procesal, de relación con los demás (Cuadro 5).

\section{Justicia sustantiva, de mínimos}

Es el conjunto de bienes precisos para alcanzar la humanidad y llevar una vida digna; el 'suelo' o base de la justicia (y la humanidad) sobre el que se puede levantar una justicia social más amplia añadiendo la distribución equitativa de otros bienes. Define un mínimo vital humano que incluiría la provisión de bienes materiales y socio-económicos (alimentación, vivienda, trabajo, salud y educación básicas), pero también, bienes psicosociales como dignidad, seguridad personal, auto-estima, autonomía personal, vinculación interpersonal, pertenencia y participación sociales.

\section{Justicia distributiva, el núcleo central}

Esta supone la idea dominante en la justicia social. La distribución equitativa de riqueza, poder, estimación y otros recursos sociales valiosos que otorgue a cada persona o grupo las mismas posibilidades de obtener los bienes materiales e inmateriales que contribuyen a su desarrollo humano. Para aclarar y aplicar la justicia distributiva es clave especificar el significado y criterios operativos de equidad en la distribución de bienes sociales y de la igualdad humana que queremos conseguir: en qué aspecto pretendemos que las personas o grupos sean iguales (lo que implicará necesariamente la desigualdad en otras cualidades o aspectos).

Los criterios de equidad sugeridos en la acción social (Doménech, 1989) son: una cantidad dada o un mínimo de bienes y recursos (el mínimo vital del punto anterior), la necesidad, el esfuerzo realizado o el mérito personal, los logros conseguidos en el trabajo y el estudio, o la igualdad de satisfacción y bienestar. La elección de cada criterio conduciría a un tipo u otro de equidad (y de justicia social) y de igualdad personal en unos aspectos a costa de desigualdades en otros. Si se da prioridad por ejemplo a los criterios de necesidad, se descuidan los de mérito o esfuerzo y se ignoran los aportes que las personas hacen a la sociedad. Si al contrario solo se considera el mérito o el logro premiando a aquellos que aportan más a la comunidad, los menos aptos -y los más vulnerables- resultarán perjudicados. Parece lógico combinar varios criterios - a menudo necesidad o vulnerabilidad y mérito o logro- para amortiguar las desigualdades generadas por la igualdad unilateralmente inducida por un solo criterio y unir los incentivos a la producción de bienes y recursos (maximizados cuando se premian esfuerzos y logros) con la redistribución de los mismos usando criterios de necesidad o vulnerabilidad.

\section{Justicia procesal o de trato}

Implica tratar a los demás y relacionarse con ellos de forma justa y equitativa. Tropezamos con la misma paradoja hallada en el tema de la equidad: isignifica eso tratar a todas las personas de la misma manera o, teniendo en cuenta el valor de la diversidad, tratar a cada uno según sus cualidades personales y circunstancias socio-culturales concretas? El criterio de tratar a todos de la misma manera en los aspectos nuclearmente humanos reconociendo aquellos que nos diferencian psicológica, social o económicamente para favorecer así a los más necesitados o desvalidos (principio de la diferencia) puede ser útil, aunque resulte probablemente insuficiente. También plantea dudas la llamada 'discriminación positiva' que supone favorecer a los grupos más vulnerables o perjudicados iContribuye esta al progreso del grupo más perjudicado y a su igualación con los otros grupos sociales o por el contrario, contribuye a perpetuar la desigualdad al no premiar el mérito sino hacer parte de un grupo excluido o maltratado (la mujer, los 'negros', los inmigrantes, etc.)?

Podemos decir en resumen, que una sociedad o comunidad es justa cuando sus miembros disponen de un mínimo de bienes materiales y psicosociales precisos para llevar una vida digna, son tratados (y se relacionan entre sí) de forma igual respetando sus legítimas diferencias socio-culturales y su 
CUADRO 5.

Justicia social: Definición y componentes

Definición: Acceso a los bienes materiales y psicosociales precisos para llevar una vida digna y valiosa, obteniendo un trato igualitario (pero que respete la singularidad psicológica y la legítima diferencia socio-cultural) y promoviendo la distribución equitativa de los bienes y recursos sociales valiosos en función de la necesidad humana y la contribución personal y colectiva a la producción de esos bienes y recursos.

\begin{abstract}
Componentes
Justicia sustantiva, mínimo vital que garantiza aa todas las personas:

- las necesidades materiales y biofísicas: alimentación, vivienda, trabajo, salud y educación básicas

- los bienes y aspiraciones psicosocales esenciales: dignidad, seguridad y autoestima, auto-dirección, vinculación interpersonal y pertenencia social

Justicia distributiva distribución equitativa de poder y bienes sociales; igualdad de oportunidades y acceso a los bienes y recursos sociales. Aquí resulta clave el criterio de equidad elegido (tipo de igualdad promovida): mínimo universal (justicia sustantiva), necesidad, esfuerzo, mérito, logro, o una combinación de varios criterios.

Iusticia procesal, trato humanamente igual para todos (según cualidades bio-psicológicas, socio-culturales y merecimiento personal de cada quién. Por medio de esta se favorece a los más débiles o necesitados (principio de la diferencia; discriminación positiva)
\end{abstract}

Fuente: elaboración propia

singularidad psicológica, y los bienes y recursos sociales existentes se distribuyen de forma equitativa garantizando que, además de tener en cuenta el esfuerzo y mérito en la generación de esos bienes y recursos valiosos, todos tienen acceso a ellos de acuerdo a la respectiva necesidad y aspiración al desarrollo personal.

\section{El papel del practicante comunitario}

Puesto que como he señalado, la promoción de la justicia social corresponde a la sociedad en su conjunto, iqué papel le corresponde al practicante comunitario? Creo que se puede concretar en las siguientes tres tareas:

- Realizar su trabajo de acuerdo a los principios sustantivos y relacionales apuntados, garantizando la equidad relacional en los intercambios con los actores sociales.

- Denunciar las desigualdades haciendo a la comunidad o la sociedad conscientes de las situaciones y casos de injusticia que conoce a través del trabajo y la investigación.

- Asegurar que todas las personas y grupos tienen acceso a los bienes psicosociales que 'administra' el practicante comunitario con independencia de la situación social y la capacidad económica de cada persona o grupo. Esta igualdad de oportunidades o de acceso de todos a los bienes psi- cosociales descansa sobre una doble obligación: la de la profesión (aportar servicios para todos los que no puedan pagarlos en el mercado) y la de la sociedad (de reconocer la necesidad de esos bienes y servicios y remunerar su realización profesional). Lo anterior exige concienciar a los profesionales y a la sociedad de la conveniencia (inecesidad?) de servicios públicos que aporten los bienes psicosociales precisos para el desarrollo humano de todas las personas con independencia de sus cualidades bio-psicológicas y su condición socio-cultural o económica.

\section{Referencias}

Beauchamp, T., \& Childress, J. (1999). Principios de ética biomédica. Barcelona: Paidós.

Bellah, R., Madsen, W., Sullivan, W., Swidler, A., \& Tipton, S. (1989). Hábitos del corazón. Madrid: Alianza Editorial.

Bermant, G., Kelman H., \& Warwick, D. (Eds.) (1978). The ethics of social intervention. Nueva York: Halstead Press.

Davidson, W. (1989). Ethical and moral dilemmas in community psychology: Tarnishing the angel's halo. American Journal of Community Psychology, 17, 385-389.

Domenech, A. (1989). Seis conceptos de igualdad. En V. Camps, S. Giner \& F. Savater, G. Marramao 
(Eds.), L'ètica del present (p. 121-128). Barcelona: Fundación Caixa de Pensions.

Doyal, L., \& Gough, I. (1994). Teoría de las necesidades humanas. Londres: McMillan.

França-Tarragó, O. (1996). Ética para psicológos. Bilbao: Descleé de Brouwer.

Heller, K. (1989). Ethical dilemmas in community intervention. American Journal of Community Psychology, 17, 367-378.

Jeger, A., \& Slotnick, R. (1982). Community mental health and behavioral-ecology. Nueva York: Plenum.

Kofkin, J. (2003). Community Psychology. Upper Saddle River, NJ: Prentice Hall.

Laue, J., \& Cormick, G. (1978). The ethics of intervention in community disputes. En G. Bermant, $\mathrm{H}$. Kelman \& D. Warwick (Eds.), The ethics of social intervention (pp. 205-231). Nueva York: Halstead.

Montero, M. (2004). Introducción a la psicología comunitaria. Buenos Aires: Paidós.

Nelson, G., \& Prilleltensky, I. (Eds.) (2005). Community Psychology. In pursuit of liberation and well-being. London: Palgrave-McMillan.

O'Neill, P. (1989). Responsible to whom? Responsible for what? Some ethical issues in community intervention. American Journal of Community Psychology, 17, 323-341.

Prilleltensky, I. (1997). Values, assumptions, and practices: assessing the moral implications of psychological discourse and action. American Psychologist, 52, 517-535.

Prilleltensky, I. (2001). Value base praxis in community psychology: Moving toward social justice and social action. American Journal of Community Psychology, 29, 748-778.

Rappaport, J. (1977). Community psychology: values, research, and action. Nueva York: Holt, Rinehart \& Winston.
Rawls, J. (1971). A theory of justice. Cambridge, Mass.: Belknap Press.

Sánchez, A. (1999). Ética de la intervención social. Barcelona: Paidós.

Sánchez, A. (2004). The values of community psychology and community action. Sánchez \& Bobzien, Conferencia presentada en el $V$ European Congress of Community Psychology, Berlin.

Sánchez, A. (2007). Manual de psicología comunitaria. Madrid: Pirámide.

Sánchez, A. (2013). iEs posible el empoderamiento en tiempos de crisis? Repensando el desarrollo humano en el nuevo siglo. Universitas Psychologica, 12(1), 285-300.

Sánchez, A. (en prensa). The ethics of community psychology: Actors, values, options, and consequences. En M. Bond, C. Keyes \& I. Serrano (Eds.), Handbook of Community Psychology. Washington: American Psychological Association.

Sen, A. (1990). Development as capability expansion. En K. Griffin \& J Knight (Eds.), Human development and the international development strategy for the 1990s (pp. 51-54). Nueva York: McmillanUnited Nations.

Serrano, I. (1994). The power of ethics and the ethics of the powerful. American Journal of Community Psychology, 22, 1-20.

Snow, D., Grady, K., \& Goyette-Ewing, M. (2000). A perspective on ethical issues in community psychology. En J. Rappaport \& E. Seidman (Eds.), Handbook of community psychology (pp. 896-917). Nueva York: Kluwer Academia/Plenum.

Thompson, A. (1989). Guide to ethical practice in psychotherapy. Nueva York: Wiley

Winkler, M., Alvear, K., Olivares, B., \& Pasmanik, D. (2012). Querer no basta: Deberes éticos en la práctica, formación e investigación en Psicología Comunitaria. Psykhe, 21, 115-129. 